\title{
Gemtuzumab Ozogamicin/Tretinoin Regimen
}

National Cancer Institute

\section{Source}

National Cancer Institute. Gemtuzumab Ozogamicin/Tretinoin Regimen. NCI Thesaurus.

Code C161926.

A chemotherapy regimen consisting of tretinoin (all-trans retinoic acid or AT RA) and gemtuzumab ozogamicin that may be used in the treatment of acute promyelocytic leukemia (APL). 